\title{
Polyhedral Line Transversals in Space
}

\author{
David Avis and Rephael Wenger \\ School of Computer Science, McGill University, 805 Sherbrooke Street W., Montreal, \\ Canada, H3A 2 K 6
}

\begin{abstract}
Algorithms are developed for determining if a set of polyhedral objects in $R^{3}$ can be intersected by a common transversal ( $s t a b b i n g$ ) line. It can be determined in $O(n \log n)$ time if a set of $n$ line segments in space has a line transversal, and such a transversal can be found in the same time bound. For a set of polyhedra with a total of $n$ vertices, we give an $O\left(n^{4} \log n\right)$ algorithm for determining the existence of, and computing, a line transversal. Helly-type theorems for lines and segments are also given. In particular, it is shown that if every six of a set of lines in space are intersected by a common transversal, then the entire set has a common transversal.
\end{abstract}

\section{Introduction}

Let $S=S_{1}, \ldots, S_{m}$ be a set of geometric objects in $R^{d}$. We say that the objects have a $k$-transversal if they are simultaneously intersected by a $k$-dimensional flat. The question of the existence of a $k$-transversal has received considerable attention in the mathematical literature, see, for example [12] and [5]. The corresponding computational issues have been considerably less studied. However, the computation of 1-transversals, or "stabbing lines" as they are called in the computing literature, has appeared in the contexts of hidden line problems [8], set partitioning [1], and updating triangulations [10].

When $k=0$ the problem reduces to that of deciding whether the given sets have a point in common. On the theoretical side, when the sets $S_{i}$ are convex, the following theorem provides an answer to this question.

Helly's Theorem. A family of $m$ convex sets in $R^{d}$ have a common intersection point if and only if every $d+1$ of the sets have a common intersection point.

On the computational side, when the sets are polyhedral, we can find an intersection point or determine that none exist by solving a linear programming problem. 
In low dimensions this can be done efficiently using Megiddo and Dyer's technique in time proportional to the number of half-spaces required to describe the input, see [15].

When $k=1$ we call the 1-transversal a stabbing line and the problems become considerably more difficult. On the theoretical side there is no analogue of Helly's theorem. In $R^{2}$ there exist configurations of $m$ line segments with stabbing lines for each $m-1$ of the line segments but no stabbing line for all $m$ line segments [12]. Hadwiger has found an additional condition which gives a Helly-like theorem in the plane. By an oval we mean a bounded, closed convex set.

Hadwiger's Theorem. A finite or countably infinite family of disjoint ovals in $R^{2}$ admits a stabbing line if and only if there is an ordering on the ovals such that each three ovals admit a line stabber in the given order.

As it stands, Hadwiger's theorem does not provide a "good" characterization since if there is no stabbing line, the theorem does not provide an efficient way to demonstrate this, even for a finite family of sets. The theorem can, however, be turned into a "good" characterization. Consider our finite family of sets $S$. Any stabber for this family induces an ordering $\left\{i_{1}, i_{2}, \ldots, i_{m}\right\}$ and a reverse ordering $\left\{i_{m}, i_{m-1}, \ldots, i_{1}\right\}$ on the indices $1, \ldots, m$. Out of the a priori factorial number of stabbers, Katchalski et al. [14] have shown that there can be at most $\left(\begin{array}{l}m \\ 2\end{array}\right)$ such orderings. This result has been improved by Wenger [16], who reduced the upper bound to $6 m+6$ and further reduced to $2 m-2$ by Edelsbrunner and Sharir [9]. In the case of polygonal objects, these potential orderings can be efficiently computed. For each ordering, it can be determined by linear programming if in fact a stabber exists which intersects the sets in the specified order [2]. The extension of Hadwiger's theorem to higher dimensions seems a challenging task. Some results on hyperplane stabbers have been obtained by Katchalski [13] and Goodman and Pollack [11].

On the computational side very little is known about stabbing lines. In the case of $n$ line segments in the plane, Edelsbrunner et al. [8] have given an $O(n \log n)$ algorithm. Edelsbrunner [7] gives algorithms for stabbing translates of a convex polygon. Avis and Doskas [2] have given a general approach for finding $d-1$ stabbers of line segments and polyhedra in $R^{d}$, using linear programming.

In dimension $d \geq 3$ no algorithms for line stabbing have been previously presented. In this paper we develop a theory for line stabbing in $P^{3}$, threedimensional projective space. We begin by considering line stabbers of lines in $\boldsymbol{P}^{3}$ and then specialize the results to line segments. Somewhat surprisingly in view of the examples mentioned above, we find a Helly-type theorem in $R^{3}$ for lines, and for line segments providing they satisfy a "non-degeneracy" assumption. Degenerate lines or segments will be called ruled. Naturally, if all the segments lie on a 2-flat they are ruled, but there are also other situations where the segments are ruled. We give efficient algorithms for finding line stabbers of lines and line segments. Next we consider convex polyhedra in $R^{3}$. We prove an 
extremal theorem that reduces the line-stabbing problem for polyhedra to a number of line-stabbing problems for segments and points. This gives rise to a polynomial-time algorithm for finding a line stabber for polyhedra.

\section{Theoretical Results}

The stabbers of a given set of lines are most easily presented in projective space. In an earlier version of this paper, most of the results presented here were proved in real space [3]. We refer the reader to the book by Borsuk [4] for a very readable treatment of projective space and quadric surfaces. We use homogeneous coordinates, where the point $\left(x_{0}, x_{1}, x_{2}, x_{3}\right)$ in $P^{3}, x_{0} \neq 0$, corresponds to the point $\left[x_{1} / x_{0}\right.$, $\left.x_{2} / x_{0}, x_{3} / x_{0}\right]$ in $R^{3}$, and the point $\left(x_{1}, x_{2}, x_{3}\right)$ in $R^{3}$ corresponds to the point $\left(1, x_{1}, x_{2}, x_{3}\right)$ in $P^{3}$. Two lines which do not intersect in $P^{3}$ are called skewed. Let $S=\left\{L_{1}, \ldots, L_{m}\right\}$ be a set of $m$ lines in $P^{3}$. If every two lines in the set $S$ are skewed, then we call the set $S$ a skewed set.

For $u, v, w, x \in P^{3}$, we let $\operatorname{det}(u, v, w, x)$ be the determinant of the matrix with the homogeneous coordinates of $u, v, w, x$ as column vectors. Let $u$ and $v$ be the coordinates of two distinct points in $P^{3}$. The unique line through $u$ and $v$ is parametrized by $\lambda u+\mu u$ where $\lambda$ and $\mu$ vary over $R$ and either $\lambda \neq 0$ or $\mu \neq 0$. If two lines $L$ and $L^{\prime}$ parametrized by $\lambda u+\mu v$ and $\lambda^{\prime} u^{\prime}+\mu^{\prime} v^{\prime}$ intersect, then there exists $\lambda, \mu, \lambda^{\prime}, \mu^{\prime}$, not all zero, such that $\lambda u+\mu v=\lambda^{\prime} u^{\prime}+\mu^{\prime} v^{\prime}$. Equivalently, $L$ and $L^{\prime}$ intersect if and only if $\operatorname{det}\left(u, v, u^{\prime}, v^{\prime}\right)=0$.

Lemma 1. Two skew lines $L$ and $L^{\prime}$ and a point $p$ which is not on $L$ or $L^{\prime}$ admit one stabber. If $L$ and $L^{\prime}$ are parametrized by $\lambda u+\mu v$ and $\lambda^{\prime} u^{\prime}+\mu^{\prime} v^{\prime}$, then this stabber intersects $L$ at

$$
q=\operatorname{det}\left(u^{\prime}, v^{\prime}, p, v\right) u-\operatorname{det}\left(u^{\prime}, v^{\prime}, p, u\right) v
$$

Proof. There is a unique hyperplane $H$ containing $L^{\prime}$ and $p$. Since $L$ and $L^{\prime}$ are skew, $L$ intersects $H$ at exactly one point $q$. The line $L^{\prime \prime}$ through $p$ and $q$ is the unique stabber of $L, L^{\prime}$, and $q$.

The hyperplane $H$ is given by the following equation:

$$
H=\left\{x: \operatorname{det}\left(u^{\prime}, v^{\prime}, p, x\right)=0, x \in P^{3}\right\} .
$$

Let $q=\operatorname{det}\left(u^{\prime}, v^{\prime}, p, v\right) u-\operatorname{det}\left(u^{\prime}, v^{\prime}, p, u\right) v$. Since $u$ and $v$ cannot both lie on $H$, either $\operatorname{det}\left(u^{\prime}, v^{\prime}, p, v\right) \neq 0$ or $\operatorname{det}\left(u^{\prime}, v^{\prime}, p, u\right) \neq 0$ and so $q$ is a point in $P^{3}$ lying on $L$. Now $q$ lies on $H$ since

$$
\begin{aligned}
\operatorname{det}\left(u^{\prime}, v^{\prime}, p, q\right)= & \operatorname{det}\left(u^{\prime}, v^{\prime}, p, \operatorname{det}\left(u^{\prime}, v^{\prime}, p, v\right) u-\operatorname{det}\left(u^{\prime}, v^{\prime}, p, u\right) v\right) \\
= & \operatorname{det}\left(u^{\prime}, v^{\prime}, p, u\right) \operatorname{det}\left(u^{\prime}, v^{\prime}, p, v\right) \\
& -\operatorname{det}\left(u^{\prime}, v^{\prime}, p, v\right) \operatorname{det}\left(u^{\prime}, v^{\prime}, p, u\right) \\
= & 0 .
\end{aligned}
$$

Thus the stabber of $L, L^{\prime}$, and $p$ intersects $L$ at $q$. 
Lemma 2. The stabbers of three skew lines in $P^{3}$ form a quadric surface $Q$.

Proof. Let $S=\left\{L_{1}, L_{2}, L_{3}\right\}$ be a set of three skew lines in $P^{3}$ where $L_{i}$ is parametrized by $\lambda u_{i}+\mu v_{i}, u_{i}, v_{i} \in P_{3}, \lambda, \mu \in R$. Let $l$ be a stabber of $S$ and let $p$ be a point on $l$ which does not lie on $L_{1}$ or $L_{2}$ or $L_{3}$. By Lemma $1, l$ intersects $L_{1}$ at

$$
q=\operatorname{det}\left(u_{2}, v_{2}, p, v_{1}\right) u_{1}-\operatorname{det}\left(u_{2}, v_{2}, p, u_{1}\right) v_{1}
$$

and hence

$$
\begin{aligned}
\operatorname{det}\left(u_{3}, v_{3}, p, q\right)= & \operatorname{det}\left(u_{3}, v_{3}, p, \operatorname{det}\left(u_{2}, v_{2}, p, v_{1}\right) u_{1}-\operatorname{det}\left(u_{2}, v_{2}, p, u_{1}\right) v_{1}\right) \\
= & \operatorname{det}\left(u_{3}, v_{3}, p, u_{1}\right) \operatorname{det}\left(u_{2}, v_{2}, p, v_{1}\right) \\
& -\operatorname{det}\left(u_{3}, v_{3}, p, v_{1}\right) \operatorname{det}\left(u_{2}, v_{2}, p, u_{1}\right)
\end{aligned}
$$

Since $l$ intersects $L_{3}, \operatorname{det}\left(u_{3}, v_{3}, p, q\right)=0$ and

$$
\operatorname{det}\left(u_{3}, v_{3}, p, u_{1}\right) \operatorname{det}\left(u_{2}, v_{2}, p, v_{1}\right)-\operatorname{det}\left(u_{3}, v_{3}, p, v_{1}\right) \operatorname{det}\left(u_{2}, v_{2}, p, u_{1}\right)=0
$$

If $p$ lies on $L_{1}$ or $L_{2}$ or $L_{3}, p$ also satisfies equation (1). Equation (1) has degree two and defines a quadric surface $Q$, so if $p$ lies on a stabber of $S$ then $p$ must lie on this quadric surface.

We claim that for every point $p$ on this quadric surface, there are stabbers of $S$ which contain $p$. If $p$ lies on $L_{1}$, then by Lemma 1 there is a stabber of $S$ and $p$. If $p$ does not lie on $L_{1}$, then by Lemma 1 there is some point $q$ on $L_{1}$ such that the line through $p$ and $q$ stabs $L_{1}$ and $L_{2}$. Since $p$ lies on the quadric surface defined above, $\operatorname{det}\left(u_{3}, v_{3}, p, q\right)=0$ and this stabber of $L_{1}, L_{2}$, and $p$ also stabs $L_{3}$. Therefore, the stabbers of $S$ form a quadric surface.

Any line which does not lie on a quadric surface intersects the quadric surface in at most two points. Thus any four skew lines have at most two stabbers or an infinite number of stabbers. It is easy but tedious to check that this statement is true for any four lines which are not skew.

We refer to a set of skew lines that admit an infinite number of stabbers as ruled. The terminology derives from the fact that the set of stabbing lines forms a doubly ruled surface. A set of three skewed lines is trivially ruled. The surface can be partitioned into two sets of lines: every pair of lines from the same set is skewed; every pair of lines from different sets is intersecting. There is thus an obvious duality between the stabbing lines and the lines to be stabbed. We will maintain the convention of upper case " $L$ " for the original data lines, and lower case "l" for computed stabbing lines.

Lemma 3. For $m \geq 3$ let $S=\left\{L_{1}, L_{2}, \ldots, L_{m}\right\}$ be a ruled set of lines lying on the quadric surface $Q$ formed by the stabbers of $L_{1}, L_{2}, L_{3}$. For every point $P \in Q$ there is a unique stabbing line through p that intersects each line in $S$. 
Proof. We prove the lemma by induction. If $m=3$ the lemma is true by Lemma 2. For $m \geq 4$, assume the lemma is true for $m-1$. Let $p$ be a point on $Q$. By Lemma 1 there is a unique stabber $l$ of $L_{1}, L_{2}$, and $p$. By the inductive assumption there is a unique stabber $l^{\prime}$ of $\left\{L_{1}, L_{2}, \ldots, L_{m-1}\right\}$ and $p$. Since $l^{\prime}$ stabs $L_{1}, L_{2}, L_{3}$, and $p$ and $l$ is the unique stabber of $L_{1}, L_{2}, L_{3}$, and $p, l^{\prime}=l$. Similarly, there is a unique stabber $l^{\prime \prime}$ of $\left\{L_{1}, L_{2}, \ldots, L_{m-2}, L_{m}\right\}$ and $p$, so $l^{\prime \prime}=l$. Thus $l=l^{\prime}=l^{\prime \prime}$ and $l$ is the unique stabber of $S$ and $p$.

Let $S=\left\{L_{1}, L_{2}, L_{3}\right\}$ and let $L_{i}$ be parametrized by $\lambda u_{i}+\mu v_{i}$. Given the quadric surface $Q$ formed by the stabbers of $S$, we can define a function $F$ which maps every point $p$ on $Q$ to the unique point $q$ on $L_{1}$ such that the line $l(p, q)$ through $p$ and $q$ is a stabber of $S$. Formally,

$$
F(p)= \begin{cases}\operatorname{det}\left(u_{2}, v_{2}, p, v_{1}\right) u_{1}-\operatorname{det}\left(u_{2}, v_{2}, p, u_{1}\right) v_{1}, & p \in Q-L_{2}, \\ \operatorname{det}\left(u_{3}, v_{3}, p, v_{1}\right) u_{1}-\operatorname{det}\left(u_{3}, v_{3}, p, u_{1}\right) v_{1}, & p \in L_{2}\end{cases}
$$

It follows from Lemmas 1 and 2 that $F$ performs the function described above. In the sequel we will need the following fact about $F(p)$.

Lemma 4. Fis a continuous function from $Q$ to $L_{1}$.

Proof. For every point $p \in Q-L_{2}$ we can define a neighborhood $N(p)$ which does not intersect $L_{2}$. For every point $p^{\prime} \in N(p)$

$$
F\left(p^{\prime}\right)=\operatorname{det}\left(u_{2}, v_{2}, p^{\prime}, v_{1}\right) u_{1}-\operatorname{det}\left(u_{2}, v_{2}, p^{\prime}, u_{1}\right) v_{1}
$$

Note that if $p^{\prime} \in L_{1}$, this formula sets $F\left(p^{\prime}\right)=p^{\prime}$. Thus $F$ is continuous at $p$. Now assume $p \in L_{2}$, and consider a neighborhood $N(p)$ small enough to be disjoint from $L_{3}$. If we first apply Lemma 1 with $L=L_{1}$ and $L^{\prime}=L_{2}$ and then reapply it with $L^{\prime}=L_{3}$, we see that formulas (2) and (3) agree up to a nonzero multiple for any point not on either $L_{2}$ or $L_{3}$. Therefore, for any $q \in N(p), F(q)$ is given by formula (3) and is therefore continuous in this region. Again $F$ is continuous at $p$.

Applying Lemma 4, if $M$ is a line segment on $Q$ with endpoints $p$ and $q$, then $F(M)$ is a line segment on $L_{1}$ with endpoints $F(p)$ and $F(q)$. Note that a line segment in projective space may correspond to either a segment or two half-lines in real space.

If $P$ is a polyhedron which intersects $Q$, then $F(Q \cap P)$ is a closed set in $L_{1}$ and is composed of the union of line segments in $L_{1}$. If $N$ is an open set inside $P$, then $F(Q \cap N)$ is an open set in $L_{1}$. Therefore, the endpoints of the line segments forming $F(Q \cap P)$ must correspond to stabbers which do not intersect the interior of $P$. These stabbers must pass through some edge of $P$.

As a consequence of Lemmas 1 and 2 we have the following Helly-like theorem.

Theorem 1. A set of $m \geq 6$ lines in $P^{3}$ have a stabbing line if and only if every six of the lines has a stabbing line. 
Proof. Let $S=\left\{L_{1}, \ldots, L_{m}\right\}$ denote the set of lines. Assume at first that they are skewed. If some set of four lines admits a unique stabber, then the conclusion is immediate. Suppose next that some set of four lines, say $L_{1}, L_{2}, L_{3}, L_{4}$, admits exactly two stabbing lines $l_{1}$ and $l_{2}$. If neither is a stabber for $S$, then there is some line $L_{i}$ missed by $l_{1}$ and some line $L_{j}$ missed by $l_{2}$. But this is impossible, since then there would be no stabber of $L_{1}, L_{2}, L_{3}, L_{4}, L_{i}, L_{j}$, a contradiction. There remains the case that each set of four lines admits an infinity of stabbers. But in this case it follows from Lemma 2 that all of the lines must lie in a quadric surface $Q$. Any line in this surface that intersects three of the lines must intersect all of them.

Now suppose that the lines are not skewed. Two lines, say $L_{1}$ and $L_{2}$, intersect at point $p$. If all the lines contained point $p$, the conclusion is immediate, so assume some line $L_{3}$ does not contain $p$. Let $H$ denote the plane containing $L_{1}$ and $L_{2}$, let $H^{\prime}$ denote the plane containing $L_{3}$ and $p$, and let $l$ be the line $H \cap H^{\prime}$. If all the lines lie in plane $H$ or $H^{\prime}$ or intersect $l$, then $l$ is a stabbing line for $S$. Otherwise some line $L_{4}$ does not lie in $H$ or $H^{\prime}$ and does not intersect $l . L_{1}, L_{2}$, $L_{3}, L_{4}$ have at most two stabbers so we can proceed as above. The theorem follows.

The theorem generalizes to all dimensions. We call a set of line segments collinear if one line contains all the line segments. A set of line segments is coplanar if the set of lines containing the line.segments can be embedded in a plane. Finally, a set of line segments is skewed if the set of lines containing the line segments is skewed and a set of line segments is ruled if the set of lines containing the line segments is ruled. Theorem 1 has the following corollary.

Corollary 1. A set of $m \geq 6$ skewed segments in $R^{3}$ that are not ruled have a stabbing line if and only if every six segments has a stabbing line.

We now turn to the problem of stabbing lines for convex polyhedra in $R^{3}$. Let $S=\left\{P_{1}, \ldots, P_{m}\right\}$ be a set of disjoint polyhedra in $R^{3}$. Using the previous lemmas we can prove the following "extremal" theorem for polyhedra. For points $x$ and $y$ in space, let $l(x, y)$ denote the line through $x$ and $y$.

Theorem 2. $S=\left\{P_{1}, \ldots, P_{m}\right\}, m \geq 2$, has a stabbing line if and only if there exists a stabbing line through:

(a) two vertices in two distinct $P_{i}$; or

(b) one vertex and two skewed edges in three distinct $P_{i}$; or

(c) two coplanar edges which are not collinear; or

(d) three skew edges.

Proof. We begin with the following:

Observation. Let $S^{\prime}=Q_{1}, \ldots, Q_{m}$ be a set of $m \geq 2$ disjoint convex polygons in the plane that admit a stabbing line. There exist indices $1 \leq r<s \leq m$ and vertices $x_{r} \in Q_{r}$ and $x_{s} \in Q_{s}$ such that $l\left(x_{r}, x_{s}\right)$ is a stabbing line for $Q_{1}, \ldots, Q_{m}$.

To see this, let $l$ be a stabber for $S^{\prime}$. By translating $l$, if necessary, we can assure 
that it goes through some vertex $x_{r}$ of some $Q_{r}$. We can rotate $l$ about $x_{r}$, maintaining the property that it is a stabber, until it passes through a vertex $x_{s}$ of some other $Q_{s}$.

Assume there is a stabbing line of $S$. There are two cases.

Case 1. There exists a stabbing line $l$ of $S$ which passes through a vertex of some polyhedron in $S$.

Assume $l$ passes through the vertex $x_{r}$ of polyhedron $P_{r}$. By rotating $l$ around $x_{r}$ in any direction, we can find a stabbing line $l^{\prime}$ which passes through $x_{r}$ and an edge $e_{s}$ of some polyhedron $P_{s}, s \neq r$. Let $H$ be the plane containing $x_{r}$ and $e_{s}$, let $Q_{r}=x_{r}, Q_{s}=e_{s}$, and $Q_{i}=H \cap P_{i}, i \neq r, s$. By the above observation there exists a stabbing line $l^{\prime}$ through two vertices $x_{r}$ and $x_{i} \in Q_{t}$. If $x_{t}$ is a vertex of $P_{t}$ then we have found a stabbing line through two vertices, satisfying condition (a). If $x_{t}$ is not a vertex of $P_{t}$, then it must lie on some edge $e_{t}$ which is not contained in $H$ and we have found a stabbing line though one vertex and two skew edges, satisfying condition (b).

Case 2. No stabbing line of $S$ passes through a vertex of any polyhedron in $S$.

By translating the stabbing line of $S$ in any direction we can find a stabbing line $l$ which intersects an edge $e_{r}$ of some $P_{r}$. Let $H$ be the plane containing $l$ and $e_{r}$, let $Q_{r}=e_{r}$, and let $Q_{i}=H \cap P_{i}, i \neq r$. By the above observation there exists a line $l^{\prime}$ through vertices $x_{s} \in Q_{s}$ and $x_{t} \in Q_{t}$, where indices $r, s$, and $t$ are distinct. For if, say, $r=s$, then there would be a stabber through a vertex of $P_{r}$ which was forbidden by assumption.

Let $x_{s}$ and $x_{t}$ lie on edges $e_{s}$ and $e_{t}$ of $P_{s}$ and $P_{i}$, respectively. By assumption $l^{\prime}$ does not intersect the endpoints of $e_{s}$ or $e_{f}$, so $e_{s}$ and $e_{t}$ do not lie in $H$ and are not collinear. If $e_{s}$ and $e_{f}$ are co-planar, then condition (c) is satisfied. Otherwise, $e_{r}, e_{s}, e_{r}$ form three skew edges and condition (d) is satisfied.

\section{Algorithms}

The algorithms follow quite naturally from the theory developed in the preceding section. Given a set $S=\left\{M_{1}, M_{2}, \ldots, M_{n}\right\}$ of $n$ nonintersecting line segments in $R^{3}$, we can find a stabber of $S$ in $O(n \log n)$ time.

If any two line segments of $\left\{M_{1}, M_{2}, M_{3}\right\}$ lie in the same plane $H$, then a stabber of $S$ must lie in $H$. We can apply the algorithm of Edelsbrunner et al. [8] for stabbing line segments in the plane in $O(n \log n)$ time. Otherwise, embed $R^{3}$ in $P^{3}$ and let $L_{1}, L_{2}, L_{3}$ be the projective lines containing $M_{1}, M_{2}, M_{3}$, respectively. By assumption these lines are skew, so the stabbers of these lines form a quadric surface $Q$. We can check in constant time whether each $M_{i}$ lies on $Q$. If some $M_{i}$ does not lie on $Q$, then it intersects $Q$ in at most two points. By Lemma 3 there are at most two stabbers of $M_{1}, M_{2}, M_{3}$, and $M_{i}$. We can check whether these stabbers stab $S$ in $O(n)$ time.

Finally, if all $M_{i}$ lie on $Q$, then we can define the function $F$ as in Lemma 4 which maps every point of $Q$ onto $L_{1}$ such that the line from $p$ to $F(p)$ is a stabber of $L_{1}, L_{2}$, and $L_{3}$. By sorting the endpoints of the line segments $F\left(M_{i}\right)$, 
we can find the intersection of all the line segments $F\left(M_{i}\right)$ in $O(n \log n)$ time. To each point in that intersection there corresponds a line stabber of $S$.

Let $S=\left\{P_{1}, \ldots, P_{m}\right\}, m \geq 2$, be a set of disjoint polyhedra in $R^{3}$. Let $n$ be the total number of vertices and edges in $S$. We will show how to find a line stabber for $S$ in $O\left(n^{4} \log n\right)$ time.

Define $F$ as in Lemma 4 and let $\boldsymbol{P}$ be any polyhedra with $t$ edges which has been preprocessed using the techniques given by Dobkin and Kirpatrick in [6] for fast reporting of polyhedral intersections. We show that if $Q$ is a quadric surface formed by the stabbers of the skew lines $L_{1}, L_{2}, L_{3}$, then we can construct $F(Q \cap P)$ in $O(t \log t)$ time. Let $T=\{F(Q \cap e): e$ is an edge of $P$ which does not lie in $Q\} . T$ is composed of at most $2 t$ points on $L_{1}$. For each point $y \in T$ store $E_{y}=\{e: y \in F(Q \cap e)$ and $e$ is an edge of $P$ which does not lie in $Q\}$. Sort the points of $T$. These points divide $L_{1}$ into at most $2 t$ line segments, each of whose interiors is either contained in $F(Q \cap P)$ or in $L_{1}-F(Q \cap P)$. For every such line segment $M_{i}$, determine if the interior of $M_{i}$ is in $F(Q \cap P)$ by choosing some point $x_{i}$ from the interior of $M_{i}$ and querying whether the unique stabber of $x, L_{2}$, and $L_{3}$ also stabs $P . F(Q \cap P)$ is the union of all the line segments $M_{i}$ whose interior lies in $F(Q \cap P)$ and the points in $T$.

Since $P$ has been preprocessed using the techniques in [6], it takes $O(\log t)$ time to find if a line intersects $P$. There are at most $2 t$ such queries. Sorting takes $O(t \log t)$ time so the total complexity for this algorithm is $O(t \log t)$.

To find a line stabber for the polyhedra in $S$ we first preprocess the polyhedra as in [6]. We then test for the four possible cases in Theorem 2:

(a) For every two vertices in $P_{j}, P_{k}, j, k$ distinct, do:

Check if the line through the two vertices stabs all the polyhedra.

(b) For every vertex and two skew edges in $P_{j}, P_{k}, P_{l}, j, k, l$ distinct, do:

Find the stabber of the vertex and two edges if it exists and check if it stabs all the polyhedra.

(c) For every two edge in $\boldsymbol{P}_{j}, \boldsymbol{P}_{k}, j, k$ distinct, which are coplanar, do:

Let $H$ be the plane containing the two edges. Intersect each of the polyhedra with $H$ to form $m$ polygons and use the algorithm of Edelsbrunner $e t$ al. [8] to find any stabbers of $S$ which lie in $H$.

(d) For every three skew edges in $P_{j}, P_{k}, P_{l}, j, k, l$ distinct, do:

Embed $R^{3}$ in $P^{3}$ and let $L_{1}, L_{2}, L_{3}$ be the projective lines containing the three skew edges. Let $Q$ be the quadric surface formed by the stabbers of these lines. Define $F$ as in Lemma 4. Apply the algorithm above to construct $F\left(Q \cap P_{i}\right)$ for each $i$. Let $T$ be the set of all the endpoints of segments in $F\left(Q \cap P_{i}\right), i=1, \ldots, m . T$ is a collection of at most $2 n$ points. Sort the points in $T$. By scanning the points in $T$ in order, intersect all the $F\left(Q \cap P_{i}\right)$. A point in this intersection has a unique stabbing line which stabs all the elements in $S$.

Preprocessing all the polyhedra takes $O\left(n^{2}\right)$ time. The total time of this algorithm is dominated by the last step. This step takes a total of $O(n \log n)$ time to construct $F\left(Q \cap P_{i}\right)$ for all $i$. It also takes $O(n \log n)$ time to sort $T$. The last step is executed $O\left(n^{3}\right)$ times for a total of $O\left(n^{4} \log n\right)$-time complexity. 


\section{Conclusion}

We presented an algorithm for finding a line transversal in $R^{3}$. We are interested in generalizing this algorithm to finding $k$-transversals in $R^{d}$. The algebraic relationships between polyhedra and their stabbers become more complex in higher dimensions and we may need tools for algebraic geometry. We know of no nontrivial lower bounds for finding transversals, even in the plane. Finally, we believe that the time complexity for finding line transversals in $R^{3}$ can almost certainly be reduced.

\section{Acknowledgment}

We would like to thank Tom Shermer for his help and suggestions on this paper.

\section{References}

1. Avis, D., Diameter Partitioning, Discrete and Computational Geometry, Vol. 1, pp. 265-276, 1986.

2. Avis, D. and Doskas, M., Algorithms for High-Dimensional Stabbing Problems, Technical Report SOCS 87.2, McGill University, Montreal, January 1987.

3. Avis, D. and Wenger, R., Algorithms for Line Stabbers in Space, Proceedings of the 3rd ACM Conference on Computational Geometry, pp. 300-307, Waterloo, 1987.

4. Borsuk, K., Multidimensional Analytic Geometry, Polish Scientific Publishers, Warsaw, 1969.

5. Danzer, L., Grunbaum, B, and Klee, V., Helly's Theorem and Its Relatives, Proceedings of Symposia in Pure Mathematics, pp. 100-181, American Mathematical Society, Providence, R1, 1963.

6. Dobkin, D. and Kirpatrick, D., Fast Detection of Polyhedral Intersection, Theoretical Computer Science, Vol. 27, pp. 241-253, 1983.

7. Edelsbrunner, H., Finding Transversals for Sets of Simple Geometric Figures, Theoretical Computer Science, Vol. 35, pp. 55-69, 1985.

8. Edelsbrunner, H., Maurer, H. A., Preparata, F. P., Rosenberg, A. L., Welzl, E., and Wood, D., Stabbing Line Segments, BIT, Vol. 22, pp. 274-281, 1982.

9. Edelsbrunner, H. and Sharir, M., The Maximum Number of Ways to Stab $n$ Convex Nonintersecting Objects in the Plane is $2 n-2$, Discrete and Computational Geometry, to appear.

10. ElGindy, H. and Toussaint, G. T., Efficient Algorithms for Inserting and Deleting Edges from Triangulations, Proceedings of the International Conference on Foundations of Data Organization, Kyoto, May 22-24, 1985.

11. Goodman, J. E. and Pollack, R., Hadwiger's Transversal Theorem in Higher Dimensions, Journal of the American Mathematical Society, Vol. 1, to appear.

12. Hadwiger, H., Debrunner, H., and Klee, V., Combinatorial Geometry in the Plane, Holt, Rinehart, and Winston, New York, 1964.

13. Katchaiski, M., Thin Sets and Common Transversals, Journal of Geometry, Vol. 14, pp. 103-107, 1980.

14. Katchalski, M., Lewis, T., and Zaks, J., Geometric Permutations for Convex Sets, Discrete Mathematics, Vol. 54, pp. 271-284, 1985.

15. Preparata, F. P. and Shamos, M. I., Computational Geometry, Springer-Verlag, New York, 1985.

16. Wenger, R., Upper Bounds on Geometric Permutations, Discrete and Computational Geometry, to appear.

Received June 14, 1987, and in revised form October 26, 1987. 